\title{
Optimal KAATSU pressure increases muscle activities during low-load exercise in an elderly female frail pa- tient receiving transcatheter aortic valve implantation
}

\author{
Ishizaka $H^{1)}$, Uematsu $A^{3)}$, Takahashi $R^{1)}$, Ogawa $H^{6)}$, Shibasaki ${ }^{6)}$, Sawaguchi $T^{2)}$, Nasuno $T^{2)}$, Toyoda \\ $S^{2)}$, Yamaguchi $S^{2)}$, Yasuda $T^{4)}$, Sato $Y^{5)}$, Fukuda $H^{6)}$, Inove $T^{2)}$, Nakajima $T^{2)}$
}

Int. J. KAATSU Training Res. 2019; 15: 5-8

Correspondence to Dr. Ishizaka H, Department of Rehabilitation, Dokkyo Medical University, 880 Kitakobayashi, Mibu, Tochigi, Japan 321-0293

E-mail: i-hayato@dokkyomed. ac.jp

Running head: EMG during KAATSU training in a frail patient

See end of article for authors' affiliations

\begin{abstract}
[Purpose] KAATSU training is a method that increases muscle activity even at low loads. In this study, we examined how different KAATSU pressure modulates elbow flexor activities during lowload elbow flexion and extension in an elderly female frail patient.

[Method] A 89-year-old female patient who had repeated hospitalization due to chronic heart failure and undergone transcatheter aortic valve implantation (TAVI) for severe aortic stenosis participated in this study. The participant performed a series of right elbow flexion and extension with a $500 \mathrm{~g}$ weight wound around the right wrist joint and surface electromyograms (EMG) of right biceps brachii (BB) and brachioradialis (BR) were recorded. The exercises conditions were in control (without KAATSU, KAATSU 0), 120 SKU (KAATSU 120) and 150 SKU (KAATSU 150) on the proximal part of right upper limb. The number of exercises was 20 trials $\times 3$ sets for each condition. We recorded muscle strength of maximum isometric voluntary contraction (MVC) measured with a hand-held dynamometer before the start of each condition. EMG was expressed as \%MVC and averaged for flexion and extension phase in each condition. A rate of perceived exertion (RPE) after each set was measured by the Borg Scale.

[Results] There was no change in muscle strength during MVC before each condition. Compared with KAATSU 0, EMG activity of both BB and BR increased during flexion in KAATSU 120 and 150. Under KAATSU 120, BB activity increased in the first half of the flexion phase and BR activity increased throughout the flexion phase. Although RPE under KAATSU 150 was higher than KAATSU 120, both EMG activities under pressure 150 was lower than under KAATSU 120.

[Conclusion] An elderly female frail patient who had received TAVI showed that muscle activity under KAATSU 120 was the highest during low-load elbow flexion and extension exercises, while RPE was second highest. These results suggest that an appropriate KAATSU pressure will increase muscle activity during low-load exercise while minimize an increase of participant's burden.
\end{abstract}

Key word: KAATSU training, EMG, female frail patient, TAVI

\section{Introduction}

The prevalence of chronic heart failure (CHF) increases with age, and CHF patients tend to have sarcopenia ${ }^{1)}$. Sarcopenia is associated with muscle weakness, decreased physical function, and then mortality in the elderly. Therefore, in a cardiac rehabilitation, a method which can achieve effective increase of muscle strength and mass to improve sarcopenia is needed in elderly patients with
CHF. In recent years, in addition to aerobic exercise, it has been recommended that middle-aged and elderly patients with coronary artery disease prescribe resistance training in order to improve exercise tolerance ${ }^{2)}$. Although moderate to high intensity exercise can improve muscle strength and mass in elderly ${ }^{3)}$, they are often in cases to retire a such high intensity exercise, especially in patients with heart disease. Therefore, the low-load exercise which can

\footnotetext{
1) Department of Rehabilitation, Dokkyo Medical University, Tochigi, Japan

2) Department of Cardiovascular Medicine, School of Medicine, Dokkyo Medical University, Tochigi, Japan

3) Department of Health and Sport Sciences, Premedical Sciences, Dokkyo Medical University, Tochigi, Japan

4) School of Nursing, Seirei Christopher University, Shizuoka, Japan

5) Chaiman, Center for KAATSU Research at Harvard Medical School, Massachusettsm, USA

6) Department of Cardiovascular Surgery, School of Medicine, Dokkyo Medical University, Tochigi, Japan
} 
improve muscle strength and mass is required even in such cases.

A training method which can be expectable to improve muscle strength and mass with low-load exercise is KAATSU training which was invented by Dr. Yoshiaki Sato. The novelty of KAATSU training is that a person performs low-load exercise under the conditions with the restricted muscle blood flow, by binding the proximal part of lower or upper extremities with a specially designed cuff. KAATSU training has been reported that muscle activity dramatically increases during low-load exercise compared with a natural condition ${ }^{4)}$. Patterson et al. ${ }^{5)}$ reported that, although the effect of KAATSU training to increase muscle strength and mass is weaker than high-load conventional exercise, a person who cannot perform high-load exercise such as postoperative rehabilitation, cardiac rehabilitation, inflammatory diseases, or frail elderly patients should execute KAATSU training to facilitate training effect on muscle strength and mass. Recently, because muscle activities during KAATSU training in patients with heart disease increased clearly with the load of $10 \%$ of 1 repetition maximum (RM) compared with $20 \%$ of 1 RM, we suggested that very low-load KAATSU training will be an optimal training intensity for a person with heart disease ${ }^{6}$.

Loenneke et al. ${ }^{7)}$ reported that a magnitude of muscle activity during KAATSU increases with increase of cuff pressure and there is a ceiling effect in this relationship, suggesting that appropriate cuff pressure maximizes muscle activation without maximize participant's burden. Previous studies selected 110-160 standard KAATSU unit (SKU) for increasing upper limb muscles in healthy young ${ }^{8,9)}$, but it is unclear that these cuff pressure also appropriates for elderly frail patient with heart disease. Therefore, in this study, we examined how muscle activity changes during KAATSU training with different pressures on the upper limbs in an elderly frail patient who had undergone transcatheter aortic valve implantation (TAVI) for severe aortic stenosis.

\section{Materials and method \\ 1. Subjects}

The subject was an 89-year-old woman (height $=143.7$ $\mathrm{cm}$, body weight $\left.=32.4 \mathrm{~kg}, \mathrm{BMI}=15.7 \mathrm{~kg} / \mathrm{m}^{2}\right)$. After undergoing TAVI for severe aortic stenosis, she had been repeatedly hospitalized due to the medical treatment of chronic heart failure. Her fingers were deformed due to rheumatic arthritis, and she had a history of complete atrioventricular block and taken an implantation of a pacemaker (DDD: 60-130). In addition, she also had a history of type 2 diabetes, dyslipidemia, and bronchial asthma. Her blood pressure was 93 / $52 \mathrm{mmHg}$. The left ventricular ejection fraction (EF) was 69\%, and plasma brain natriuretic peptide (BNP) was $210.4 \mathrm{pg} / \mathrm{ml}$.

Her physical functions were as follows: normal walking speed $=0.51 \mathrm{~m} / \mathrm{sec}$, right grip strength $=6.1 \mathrm{kgf}$, left grip strength $=0.0 \mathrm{kgf}$. Because she had been implanted the pacemaker, we could not measure her muscle mass by a body composition meter based on the bioelectrical impedance method.

After receiving an explanation, the Dokkyo Medical University Hospital Research Ethics Review Board approved the study protocol (approval number: 27074) which was conducted according to Declaration of Helsinki.

\section{Methods}

Figure 1 shows the apparatus of the experiment. The subject repeatedly performed the right elbow joint flexion and extension with attached $500 \mathrm{~g}$ weight belt at the wrist joint in the sitting position at a chair with knee and hip flexed $90^{\circ}$. The range of motion was from fully elbow extended to fully flexed position. The exercise cycle was consisted of each $1 \mathrm{sec}$ flexion and extension. The subject matched the exercise cycle with 60 beat-per-minute click sound which was made by an electric metronome. The experimenter counted numbers of exercise cycle matching with click sounds to minimize a variability of subject's exercise cycle.

Surface electromyography (EMG) of the right biceps brachii (BB) and brachioradialis (BR) were recorded using a surface electrode (width $2 \mathrm{~mm}$, length $10 \mathrm{~mm}$, interelectrode $10 \mathrm{~mm}$, SS-209, Nihon Kohden) placed on the belly of right $\mathrm{BB}$ and $\mathrm{BR}$. The ground electrode was attached to the skin on the right upper iliac spine. The EMG signal was submitted from the transmitter (ZB-581G, Nihon Kohden) to the receiver (ZR-550H, Nihon Kohden). The goniometer (SG 150, Biometrics) was fixed to the lateral side of the elbow joint with double-sided tape to monitor elbow joint flexion and extension. EMG and goniometer signals were synchronized, filtered (EMG: bandpass, 15$500 \mathrm{~Hz}$; goniometer: low pass, $6 \mathrm{~Hz}$ ), and sampled at 2 $\mathrm{kHz}$ using a multi signal recording system (WEB5500, Nihon Kohden).

The KAATSU system (KAATSU Nano, KAATSU Global) was used to restrict blood flow in the upper arm. A pneumatic cuff (width $60 \mathrm{~mm}$, KAATSU Air Bands, KAATSU Global) was attached to the proximal end of participant's right upper arm. We set 3 pressure intensities such as 0 (KAATSU 0), 120 (KAATSU 120), and 150 SKU (KAATSU 150).

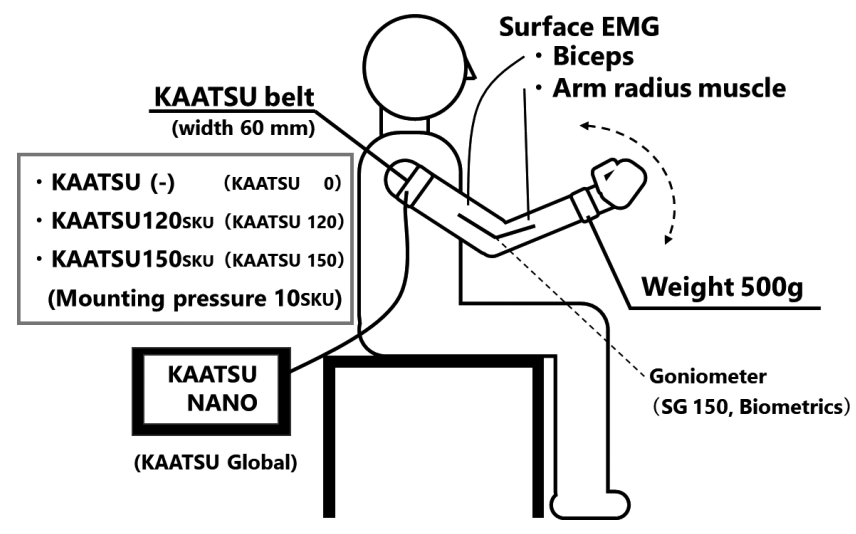

Figure 1. Measurement method 


\section{Exercise protocol}

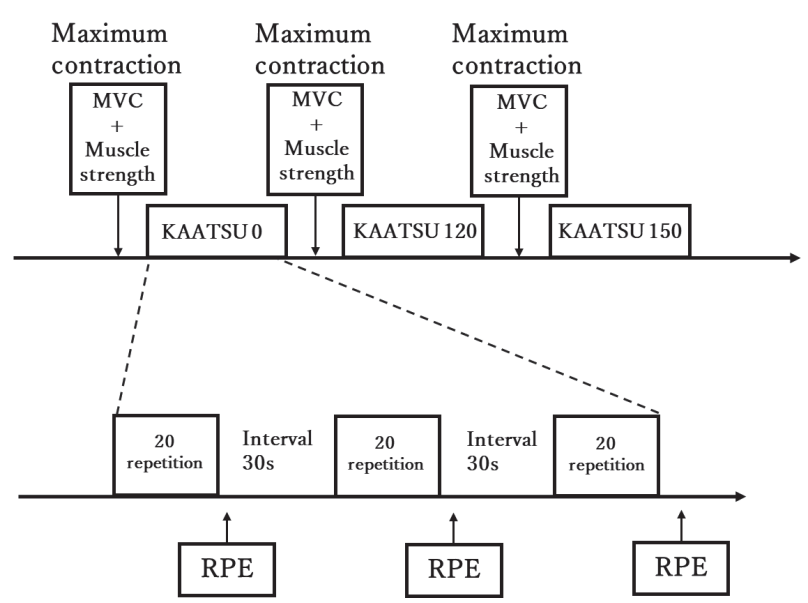

Figure 2. Exercise protocol maximum isometric voluntary contraction

Figure 2 illustrates the measurement protocol. The participant executed 3 sets of 20 trials of right elbow flexion-extension exercise in each pressure condition with 30 $s$ of rest between sets and 5 min of rest between conditions. To minimize participant's burden, experimental trials were performed in the order of KAATSU 0, 120, and 150. We measured the rate of perceived exertion (RPE) using 15 steps Borg Scale ${ }^{10)}$ after each set.

Before the start of each condition, EMG and muscle strength measured by a handheld dynamometer (Mutus F-1, Anima) during maximal isometric voluntary contraction (MVC) of right elbow joint flexion with right elbow flexed $90^{\circ}$ in the sitting position was recoded.

EMG signals were normalized as \%MVC. Each flexion and extension phase was divided into the first and second half and evaluated based on the averaged EMG for all trials in a condition.

\section{Result}

The muscle strength at the start of each condition was similar value (Table 1). KAATSU-induced EMG increase in $\mathrm{BB}$ and $\mathrm{BR}$ was greater in flexion than extension phase (Table 2, Figures 3 and 4). Compared with KAATSU 0, EMG of BB increased in the first half and EMG of BR increased in whole flexion phase at KAATSU 120. EMG of $\mathrm{BB}$ and BR at KAATSU 150 was lower than KAATSU 120. RPE was 11 for all 3 sets at KAATSU 0, but gradually increased at KAATSU 120 and 150 (Table 1).

\section{Discussion}

The present study demonstrated that, compared with no KAATSU pressure, muscle activity in BB increased in the first half and BR increased during whole of flexion phase while KAATSU pressure was moderate (KAATSU 120 ), but did not increased if KAATSU pressure was strong (KAATSU 150) in an elderly female frail patient receiving TAVI. RPE increased with increasing KAATSU pressure, suggesting that strong KAATSU pressure tends
Table 1. Isometric elbow flexor strength before exercise set and rate of perceived exertion (RPE) immediately after exercise

\begin{tabular}{lccc}
\hline & KAATSU 0 & KAATSU 120 & KAATSU 150 \\
\hline Muscle strength by HHD (kg) & 5.55 & 5.60 & 5.15 \\
RPE: Borg Scale & $11 / 11 / 11$ & $12 / 13 / 15$ & $13 / 14 / 15$ \\
\hline
\end{tabular}

HHD: Hand-held Dynamometer.

Table 2. Muscle activities (\%MVC) during exercise

\begin{tabular}{llcrrrrr}
\hline & \multirow{4}{*}{ Muscle } & \multicolumn{3}{c}{ Flexion phase } & \multicolumn{3}{c}{ Extension phase } \\
& & KAATSU 0 & KAATSU 120 & KAATSU 150 & KAATSU 0 & KAATSU 120 & KAATSU 150 \\
\hline BB & All & $29.9 \pm 2.6$ & $32.5 \pm 3.0$ & $29.2 \pm 2.3$ & $10.5 \pm 0.7$ & $12.2 \pm 1.8$ & $5.9 \pm 0.9$ \\
& First half & $18.4 \pm 4.8$ & $23.9 \pm 3.8$ & $16.5 \pm 4.6$ & $14.1 \pm 2.6$ & $17.4 \pm 2.9$ & $7.0 \pm 1.4$ \\
& Second half & $41.4 \pm 3.2$ & $41.2 \pm 3.1$ & $41.9 \pm 1.8$ & $6.6 \pm 1.3$ & $7.0 \pm 1.0$ & $4.8 \pm 1.1$ \\
BR All & $15.1 \pm 1.5$ & $26.7 \pm 4.1$ & $16.6 \pm 1.8$ & $4.9 \pm 1.1$ & $6.1 \pm 0.5$ & $5.9 \pm 0.9$ \\
& First half & $10.1 \pm 2.7$ & $18.6 \pm 4.2$ & $6.9 \pm 2.5$ & $6.6 \pm 1.9$ & $8.2 \pm 1.4$ & $7.0 \pm 1.4$ \\
& Second half & $20.0 \pm 1.6$ & $34.7 \pm 4.8$ & $26.4 \pm 2.4$ & $3.2 \pm 0.4$ & $4.1 \pm 0.6$ & $4.8 \pm 1.1$ \\
\hline Values are mean \pm SD. BB: Biceps brachii, BR: Brachioradialis. & & &
\end{tabular}

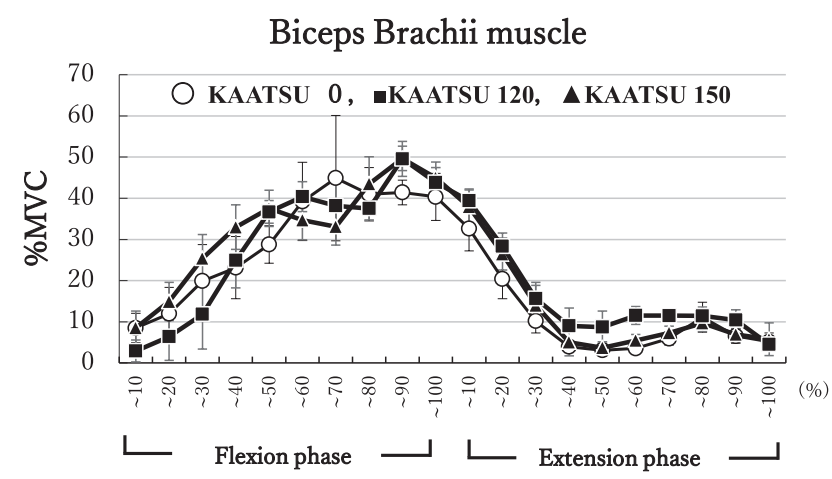

Values are mean $\pm \mathrm{SD}$

Figure 3. Changes in flexion and extension of the biceps over time

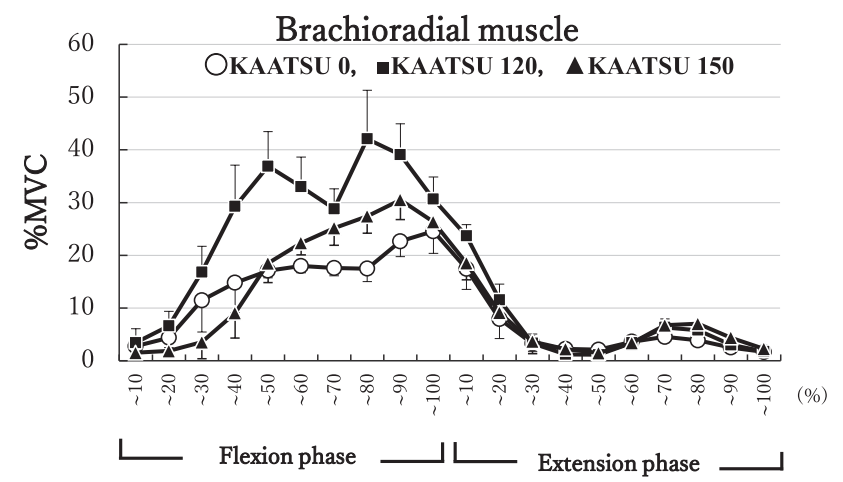

Values are mean \pm SD

Figure 4. Changes in flexion and extension of the peroneus muscle

to increase fatigue.

Loenneke et al. ${ }^{11)}$ reported that there is hormesis effect of KAATSU pressure on muscle activity and recommended that KAATSU pressure should be set at the level such that it blocks the return of the veins and allows blood to enter the muscle. In the present study, we selected 120 and $150 \mathrm{SKU}$ as KAATSU pressure based on previous studies for healthy young male ${ }^{8,9)}$ and revealed that 150 
SKU may represented hormesis effect on muscle activity in elbow flexors in an elderly female frail patient. Therefore, we speculated that optimal KAATSU pressure to increase a muscle activity depends on a personal body composition.

Ilett et al. ${ }^{12)}$ recommend that the pressure which induces $60-80 \%$ of complete arterial occlusion is appropriate for KAATSU training. She had low systolic blood pressure lower than $100 \mathrm{mmHg}$. The patient participated in this study was unable to measure grip strength due to rheumatoid arthritis and was unable to measure with a body composition monitor because she had been implanted a pacemaker. However, her slow normal walking speed $(0.51 \mathrm{~m} /$ s), low BMI $\left(15.7 \mathrm{~kg} / \mathrm{m}^{2}\right)$, and small upper arm circumference strongly suggests that her muscle mass is very low. Therefore, it is possible that, when the proximal part of the upper arm is compressed by pressurization, the effect of soft tissue to resist the compression stimulus is weak and an arterial occlusion is greater than young healthy male. Thus, a possible reason why the optimal KAATSU pressure intensity for increasing muscle activity in an elderly female frail patient was lower than that of healthy young male ${ }^{13.14)}$ depended on a body composition as well as blood pressure in the elderly frail patient. There is also a possibility that age-dependent atherosclerosis may resist to KAATSU pressure, but the effect of atherosclerosis will be minor compared to the intensity of pressurization. Future study will be needed to examine the relation between participants' body composition (e.g. BMI, muscle mass) and KAATSU pressure which increase a muscle activity.

RPE increased without enhancing effective muscle activity when the pressure intensity is too high in an elderly female frail patient with heart disease, suggesting that pressurization which exceeds an optimal intensity wrongfully increases participant's burden during exercise.

The trial order was fixed in this study, however a trial-order effect on muscle activities was weak because muscle strength before each condition was similar level. A limitation of this study is a single case report. Further data collection will be needed for similar patients. In addition, a weaker KAATSU pressure than 120 SKU will be examined whether it can increase muscle activity and not increase participant's burden. The maximal RPE was 15, suggesting that low-load KAATSU training is useful to increase muscle strength with safety and do not need excessive burden to elderly CHF patients. We strongly suggest that a therapist especially considers KAATSU pressure if not exceed optimal intensity to improve the training efficacy.

\section{Conclusions}

An elderly female frail patient who had received TAVI showed high muscle activity at 120 SKU, compared with 150 SKU during the elbow joint flexion and extension exercises. And, the setting of the appropriate KAATSU pressure is required for performing an effective and safe KAATSU training in each case.

\section{$\ldots$}

\section{References}

1) Von Haehling $\mathbf{S}$ (2018) Muscle wasting and sarcopenia in heart failure: a brief overview of the current literature. ESC Heart Fail 5: 1074-1082.

2) Yamamoto S, Hotta K, Ota E, et al. (2016) Effects of resistance training on muscle strength, exercise capacity, and mobility in middle-aged and elderly patients with coronary artery disease: A meta-analysis. J Cardiol 68: 125-134.

3) Peterson MD, Rhea MR, Sen A, et al. (2010) Resistance exercise for muscular strength in older adults: a meta-analysis. Ageing Res Rev 9: 226-237.

4) Cook SB, LaRoche DP, Villa MR, et al. (2017). Blood flow restricted resistance training in older adults at risk of mobility limitations. Exp Gerontol 99: 138-145.

5) Patterson SD, Hughes L, Warmington S, et al. (2019) Blood Flow Restriction Exercise Position Stand: Considerations of Methodology, Application, and Safety. Front Physiol 10: 533.

6) Ishizaka H, Uematsu A, Mizushima Y, et al. (2019) Blood Flow Restriction Increases the Neural Activation of the Knee Extensors During Very Low-Intensity Leg Extension Exercise in Cardiovascular Patients: A Pilot Study. J Clin Med 8: E1 252.

7) Loenneke JP, Kim D, Fahs CA, et al. (2015) Effects of exercise with and without different degrees of blood flow restriction on torque and muscle activation. Muscle Nerve 51: 713-721.

8) Yasuda T, Loenneke JP, Thiebaud RS et al. (2012) Effects of Blood Flow Restricted Low-Intensity Concentric or Eccentric Training on Muscle Size and Strength. PLoS One 7: e52843.

9) Yasuda T, Fujita S, Ogasawara R, et al. (2010) Effects of low-intensity bench press training with restricted arm muscle blood flow on chest muscle hypertrophy: a pilot study. Clin Physiol Funct Imaging 30: 338-343.

10) Borg, G. (1982) Psychophysical Bases of Perceived Exertion. Med Sci Sports Exerc 14: 377-381.

11) Loenneke JP, Thiebaud RS, Abe T, et al. (2014) Blood flow restriction pressure recommendations: The hormesis hypothesis. Med Hypotheses 82: 623-626.

12) llett MJ, Rantalainen T, Keske MA, et al. (2019) The Effects of Restriction Pressures on the Acute Responses to Blood Flow Restriction Exercise. Front Physiol 10:1018.

13) Yasuda T, Fukumura K, Fukuda T et al. (2014) Effects of low-intensity, elastic band resistance exercise combined with blood flow restriction on muscle activation. Scand J Med Sci Sports 24:55-61.

14) Yasuda T, Brechue WF, Fujita T et al. (2008) Muscle activation during low-intensity muscle contractions with varying levels of external limb compression. J Sports Sci Med 7:467-474.

Indication of KAATSU training and "KAATSU training" in the text are registered as trademarks of KAATSU JAPAN Co., Ltd.

\section{Authors' affiliations}

Ishizaka H, Takahashi R, Department of Rehabilitation, Dokkyo Medical University, Tochigi, Japan

Nakajima T, Sawaguchi T, Yamaguchi S, Nasuno H, Toyoda S, Inove T, Department of Cardiovascular Medicine, School of Medicine, Dokkyo Medical University, Tochigi, Japan

Uematsu A, Department of Health and Sport Sciences, Premedical Sciences, Dokkyo Medical University, Tochigi, Japan

Yasuda T, School of Nursing, Seirei Christopher University, Shizuoka, Japan

Ogawa H, Shibasaki I, Fukuda H, Department of Cardiovascular Surgery, School of Medicine, Dokkyo Medical University, Tochigi, Japan 